\title{
Sensation im Botanischen Garten Bogor (Indonesien): eine in Kultur blühende Rafflesia
}

\author{
HERWIG ZAHORKA
}

\begin{abstract}
Numerous grafting and inoculation experiments starting in 2004 and executed at the roots of garden situated Tetrastigma host plants finally succeeded after many failures. On June $3^{\text {rd }}, 2010$ the first parasitic Rafflesia patma flower opened far outside of its natural habitat. Seven buds appeared nearly at the same time.
\end{abstract}

\section{Zusammenfassung}

2004 begann man im Botanischen Garten Bogor unter Anwendung verschiedener Pfropf- und Okuliertechniken, an den Wurzeln kultivierter Tetrastigma-Lianen parasitische Rafflesia zu kultivieren. Nach jahrelangen Fehlschlägen erschienen Ende 2009 mehrere Knospen. Am 3. Juni 2010 öffnete sich die erste Blüte einer Rafflesia patma, die damit erstmals außerhalb ihres natürlichen Standortes zu bestaunen war.

\section{Vorgeschichte}

Im Schutzgebiet Pangandaran im südlichen West-Java fruchtete 2004 eine Rafflesia patma, von der reichlich Samen gesammelt wurden. Ein Samenansatz ist bei Rafflesien nicht selbstverständlich, denn für eine erfolgreiche Befruchtung müssen eine weibliche und eine männliche Blüte gleichzeitig erscheinen, was äußerst selten geschieht. Rafflesien sind Vollparasiten, die weder Blätter, Chlorophyll, Stängel noch Wurzeln haben. Für uns sichtbar sind nur die spektakulären Blüten, die an den Wirtspflanzen erscheinen. Sie werden gelegentlich als das größte Wunder im Pflanzenreich bezeichnet (vgl. auch ZAHORKA 2003, 2006, 2007, 2011). Ausbreitung und Vermehrung dieser extrem seltenen Pflanzen ist bis heute ein Rätsel. Ihre Wirtspflanzen sind Lianen der Gattung Tetrastigma (Vitaceae), in deren Wurzeln sie parasitieren (WHITTEN et al. 1996).

Mit den winzigen Samen und mit möglicherweise infizierten Wurzelabschnitten aus Pangadaran experimentierte man ab 2004 an Wurzeln von Tetrastigma-Lianen, die im Botanischen Garten Bogor kultiviert werden. Dabei wurden verschiedene Pfropf- und Okuliertechniken angewendet. In den Folgejahren erschienen tatsächlich einige Rafflesia-Knospen. Leider verkümmerten alle, bevor sie sich zu einer

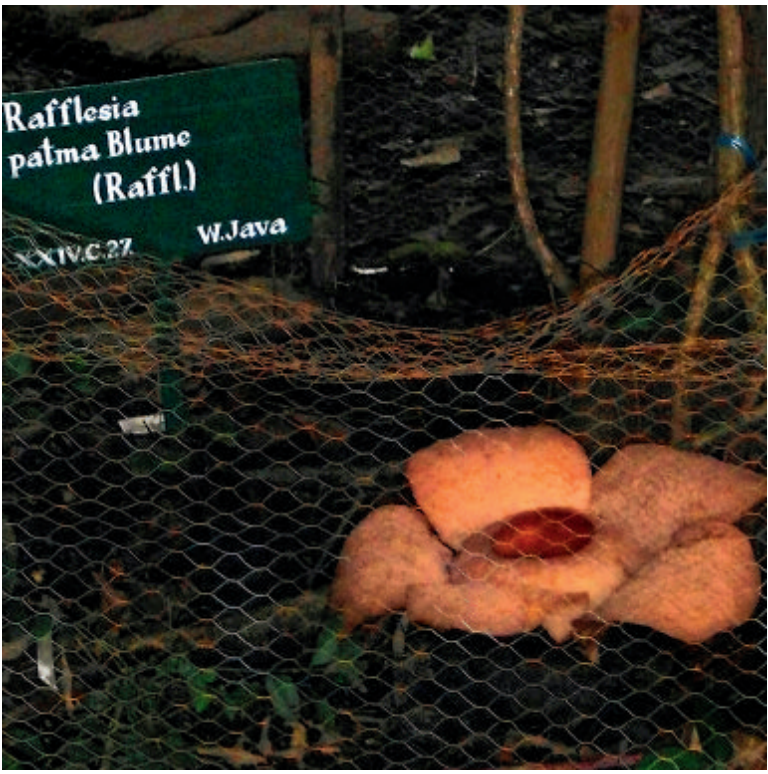

ausgewachsenen Blüte entwickeln konnten, weshalb neue Methoden ausprobiert wurden.

\section{Erfolgsgeschichte}

2008 verlegte man die Versuche in ein eingezäuntes Pflanzen-Quartier, in dem 2007 mehrere junge Exemplare von Tetrastigma cf. lanceolarium aus Pangadaran angepflanzt worden waren. Da sie kräftig wuchsen, wurden an den Wurzeln dieser Pflanzen Pfropf- und Okuliermaßnahmen durchgeführt. Nach fast zwei Jahren erschienen an den Wurzeln von drei dieser Wirtspflanzen mehrere Knospen. Dabei war nicht feststellbar, ob diese Knospenbildung durch Pfropfung bzw. 

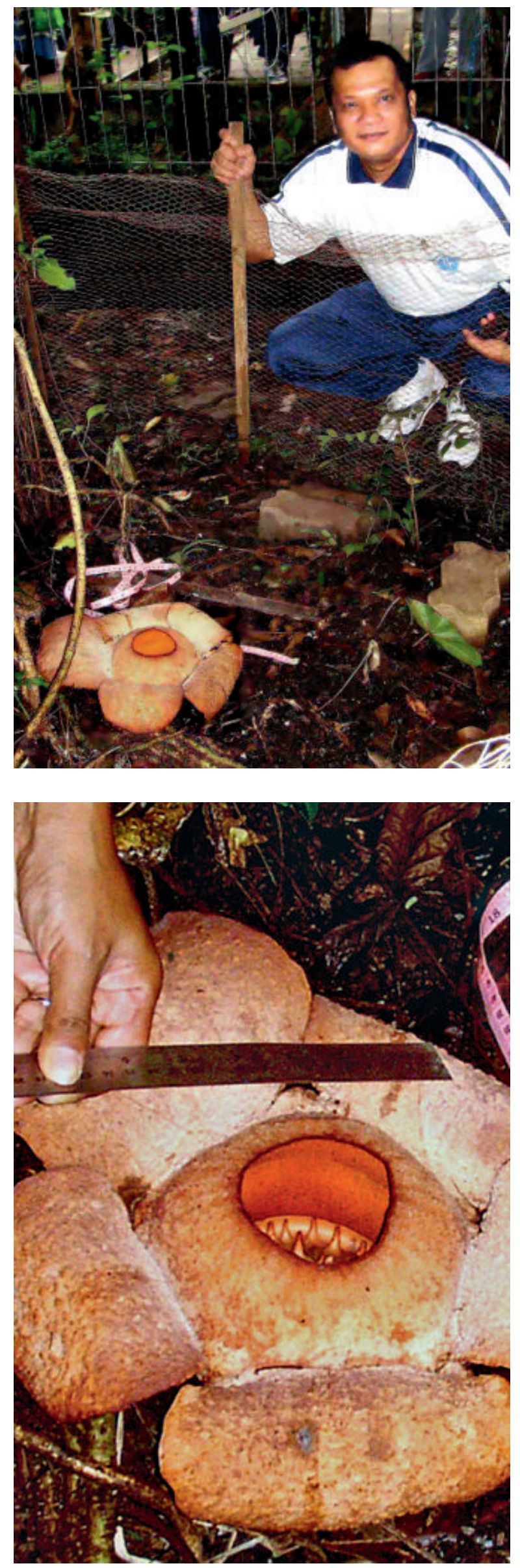

Okulation ausgelöst wurden, oder ob die eingebrachten Pflanzen bereits vorher infiziert waren. Einige Okulier-Narben befinden sich in der Nähe der Knospen, was für die erste Vermutung sprechen könnte.

Die Knospen wuchsen unterschiedlich schnell. Es dauerte einige Monate, bis sich am 3. Juni 2010 um 21 Uhr überraschend drei Perigonblätter der ersten Blüte öffneten. Am nächsten Morgen waren alle fünf Blütenblätter voll entfaltet. Dieser bisher einmalige Erfolg ist den indonesischen Wissenschaftlerinnen SOFI MURSIDAWATI, MELANIE KURNIA RisWATI und der vorherigen Direktorin des Gartens, Frau Irawati, zuzuschreiben, die seit 2004 mit großer Ausdauer und Geduld in diesem Rafflesia-Projekt arbeiten. Alle sind professionelle Orchideen-Expertinnen.

\section{Das kurze Leben der Wunderblüte}

Am 4. Juni 2010 war die lachsfarbene Blüte voll entfaltet. Sie hatte an ihrer breitesten Stelle einen Durchmesser von $38 \mathrm{~cm}$ und erreichte eine Höhe von $16 \mathrm{~cm}$. Am dritten Blühtag, dem 5. Juni, war die Farbe der prallen Blüte etwas nachgedunkelt. Um dem zahlreichen Publikum eine nahe Ansicht zu gewähren, hatte man eine Fernsehkamera neben der Blume installiert und das Bild auf einen großen öffentlichen Bildschirm, der sich außerhalb des Zauns befand, übertragen. So konnten Tausende das Blütenwunder bestaunen, ohne dabei die Rafflesia zu gefährden. Gegen Abend des 5. Juni verströmte die Blüte einen Aas-ähnlichen Gestank, wodurch grünliche Aasfliegen angelockt wurden. Bereits am 6. Juni zeigten sich an der Blüte deutliche Zerfallserscheinungen. Die Farbe veränderte sich ins Bräunliche. Helle und dunkle Fäulnisflecken traten auf, winzige ungeflügelte Termiten und Fruchtfliegen ließen sich auf den Perigonblättern nieder. Dieser Zerfallsprozess setzte sich verstärkt auch noch am nächsten Tag fort. Am sechsten Tag nach dem Aufblühen erschien die inzwischen abgestorbene und eingetrocknete Blüte in

Abb. 2 (oben): Gartendirektor MUSTAID SIREGA begutachtet die Blüte.

Abb. 3 (unten): Die Blüte hat am 4. Juni einen Durchmesser von $38 \mathrm{~cm}$ erreicht. 
dunklem Rotbraun und hatte eine fast korkähnliche Konsistenz. Im nachfolgenden schwarzen Zustand wurde sie geerntet und ins Herbarium Bogoriense zur anatomisch-morphologischen Untersuchung gebracht. Weitere sieben Knospen verschiedener Größe standen danach noch bereit und lassen auf weitere Erfolge hoffen.

\section{Männliche oder weibliche Blüte?}

Rafflesien sind zweihäusig, sie haben männliche und weibliche Blüten. Falls die Blüte im Garten männlich war, sollte zunächst versucht werden, noch an den Blütenresten verbliebenen Blütenstaub mit einem Mini-Staubsauger abzusammeln. Das hierfür vorgesehene Gerät dient normalerweise dazu, Zwischenräume zwischen den Tasten einer Computer-Tastatur zu säubern. Mit diesem Pollen könnten dann möglicherweise weibliche Blüten, die vielleicht in Zukunft im Garten erscheinen, bestäubt werden. Das Sammeln des Pollens aus Rafflesia-Blüten ist allerdings nicht einfach, da die Sexualorgane an der fast unzugänglichen Unterseite der im Inneren befindlichen scheibenförmigen Cupula in einer ringförmigen Rille untergebracht sind (vgl. Solms-Laubach 1901, Harms 1935, Meijer 1997, ZAHORKA 2006, 2007).

Der Autor versuchte deshalb, Pollen mit einem Zahnarztspiegel und einem zurecht gebogenen Plastikstäbchen, das am vorderen Ende mit etwas Baumwolle umwickelt war, aufzunehmen. Der Versuch brachte jedoch keinen Blütenstaub zutage, weshalb davon ausgegangen werden konnte, dass es sich um eine weibliche Blüte handelte. Dies bestätigte sich später bei der anatomischen Untersuchung mit der Identifizierung des Fruchtknotens mit den unbefruchteten Samenanlagen.

\section{Weitere Aussichten}

Vor über 80 Jahren begannen in Bogor niederländische Botaniker mit ähnlichen Versuchen zur

Abb. 4 (oben): Zentrum der Blüte 5 Stunden nach dem Öffnen.

Abb. 5 (Mitte): Blick in das Blüteninnere.

Abb. 6 (unten): Erste Verfallserscheinungen der Blüte sind am 6 . Juni erkennbar.
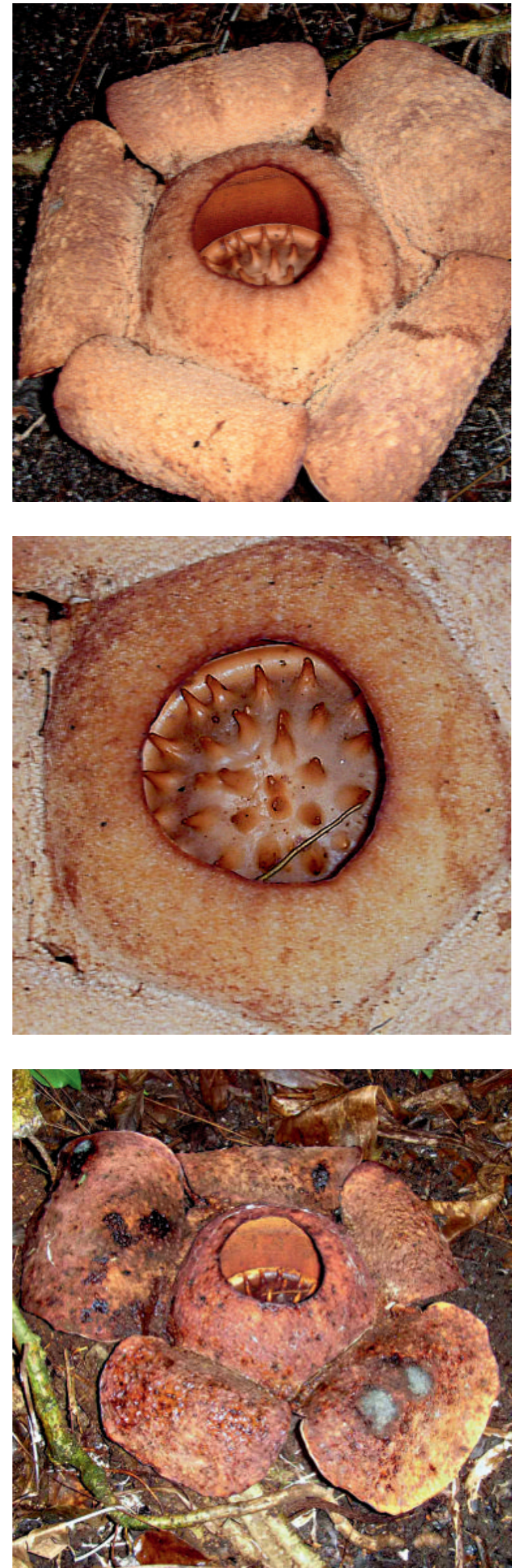

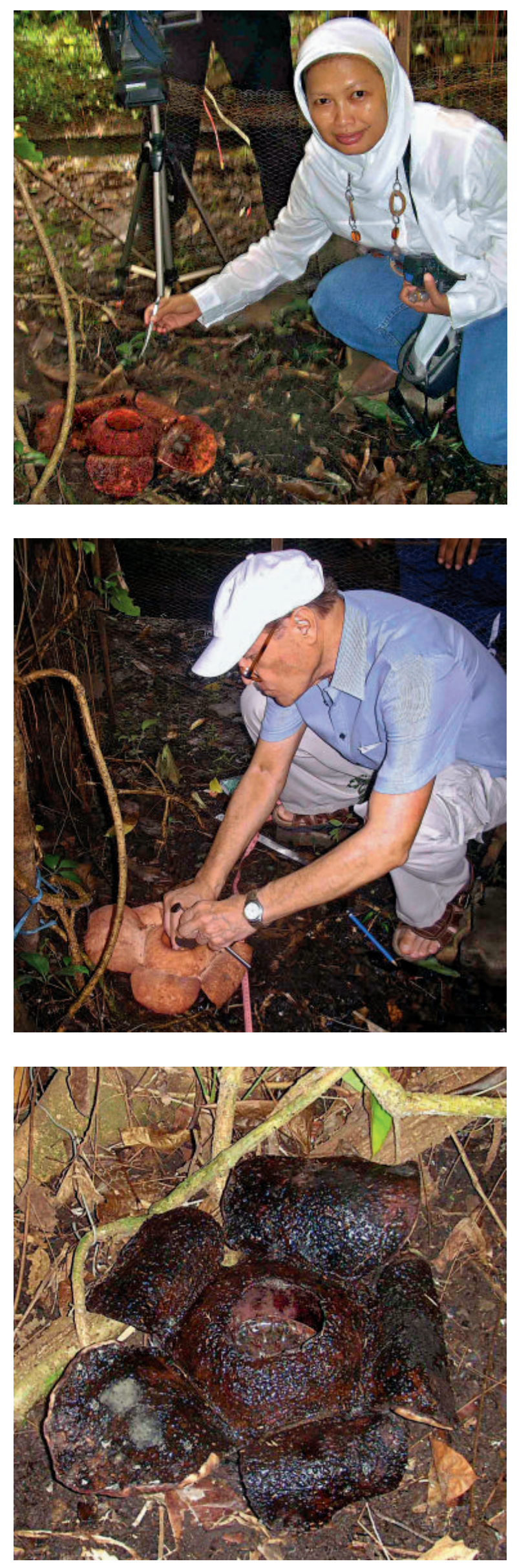

Kultur von Rafflesia. Der jetzige sensationelle Erfolg soll der erste Schritt zu erweiterten Forschungsaktivitäten sein. Die beteiligten Wissenschaftlerinnen wollen weitere Rafflesia-Populationen zur natürlichen Vermehrung im Botanischen Garten Bogor bringen. Laut SOFI MursidaWATI seien Regeneration, Erhaltung und Kultivierung dieser Pflanzen nicht unmöglich, aber eine wunderbare Herausforderung zu weiteren vertieften Studien. Sie hat mit ihrem Erfolg den Botanischen Garten in der internationalen botanischen Literatur bekannt gemacht.

\section{Schlussbemerkung}

Es würde den Botanischen Garten Bogor besonders auszeichnen, wenn sich ausgerechnet dort Rafflesia patma erfolgreich kultivieren lässt. Die Art wurde nämlich 1825 von dem aus Braunschweig stammenden deutschen Botaniker KARL LudWIG V. Blume (1796-1862) entdeckt und wissenschaftlich beschrieben. REINWARDT, der deutsche Gründer (1817) und erste Direktor des Gartens in Bogor, hatte sich V. BLumE als Mitarbeiter geholt. VON BLUME wurde später der zweite Direktor des Gartens (1822-1826), der in regem Briefwechsel mit GOETHE stand.

Nach Fertigstellung des Manuskriptes öffnete sich vom 21. bis 23. Juli 2010 eine zweite Rafflesia-Blüte. Ihr Durchmesser betrug $35 \mathrm{~cm}$, leider war sie wieder weiblich.
Abb. 7 (oben): Die Wissenschaftlerin Sofi MurSIDAWATI untersucht die Blüte.

Abb. 8 (Mitte): Der Autor an der Rafflesia-Blüte versucht Pollen zu sammeln.

Abb. 9 (unten): Am 8. Juni ist die Blüte bereits braun. Abb. 10 (S. 89): Weitere Knospen stehen bereit. 


\section{Literatur}

Harms, H. 1935: Rafflesiaceae. In: Engler, A. \& Prantl, K. (Hrsg.): Die natürlichen Pflanzenfamilien. 2. Aufl., Bd. 16 b. - Berlin.

MeIJER, W. 1997: Rafflesiaceae. In: Flora Malesiana, Bd. 13, Serie I, Seed Plants. - Leiden.

SOlms-LaUbaCH, H. 1901: Rafflesiaceae. In: EnGLeR, A. (Hrsg.): Natürliche Pflanzenfamilien, Bd. 5. - Berlin. Whitten, T., SoeriaAtmadja,R. E. \& Afiff, S. A. 1996:

The ecology of Java and Bali. - Singapur.

ZAHORKA, H. 2003: Expedition in das Innere der Insel Borneo entdeckt neuen Rafflesia-Standort. - Palmengarten 67: 19-27. ZAHORKA, H. 2006: Rafflesia - Die seltenste Blume der Welt - Das größte Wunder im Pflanzenreich. - KITA 3/06: 48-55. ZAHORKA, H. 2007: Knospen von Rhizanthes

(Rafflesiaceae) - eine spannende Entdeckung in West-

Sumatra. - Palmengarten 71: 132-134.

ZAHORKA, H. 2011: New Site of Rhizanthes infanticida

BAENZIGER et HANSEN SP.N. (Rafflesiaceae) was discovered in West-Sumatra. - The Indonesian Botanic Gardens Bulletin - Bull. Kebun Raya Ind. (im Druck).

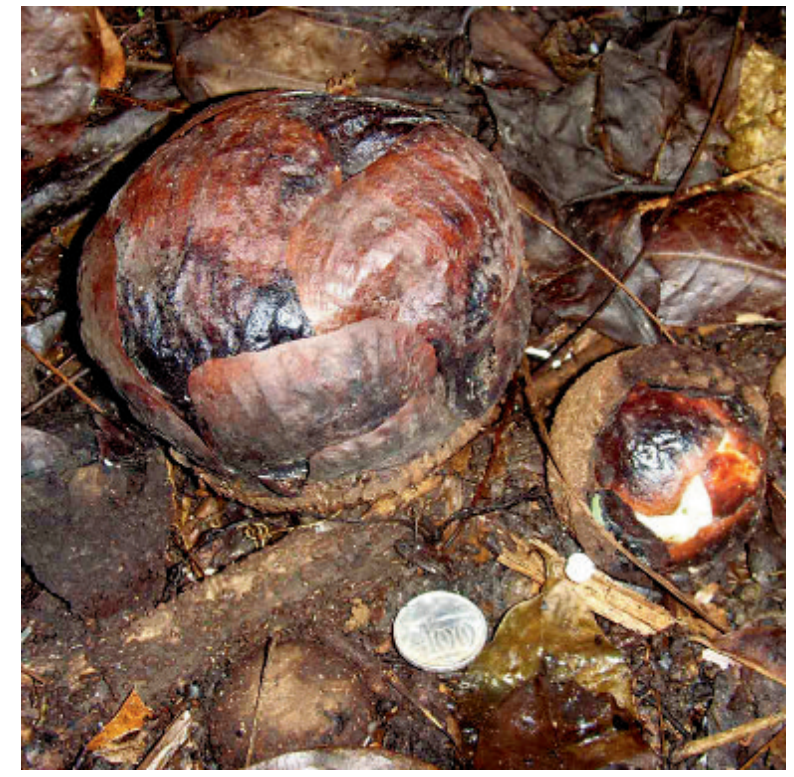

\section{Gärtnerisch-botanische Literatur}

\section{BARBARA VOGT}

\section{SIESMAYERs Gärten}

Societäts-Verlag, Frankfurt am Main, 2009, 96 Seiten, zahlreiche farbige und schwarz-weiße Abbildungen, broschiert, 12,80 Euro;

ISBN 978-3-7973-1151-1.

Mit diesem Buch ist der Herausgeberin, der KulturRegion FrankfurtRheinMain, ein schöner Start in eine neue Reihe über Gärten, Parks und Grünanlagen im Rhein-Main-Gebiet gelungen. Erfreulicherweise konnte als Autorin BARBARA VOGT, eine der fundiertesten Kennerinnen des Werkes und Lebens Heinrich Siesmayers, gewonnen werden.

Im Frankfurter Raum ist SIESMAYER vermutlich vor allem als Begründer des Palmengartens bekannt, liegt er ja auch an der Siesmayerstraße. Seit der Landesgartenschau in Bad Nauheim ist auch der dortige Kurpark, ebenfalls ein Werk SIESMAYERs, stärker ins Bewussstsein gerückt.

Im vorliegenden Band werden SIESMAYER, seine Familie und seine Gartenschöpfungen vorgestellt. Zunächst werden kurz SIESMAYERs Kindheit und beruflicher Werdegang aufgezeichnet.
Dabei werden die Texte durch Zitate SiESMAYERs aufgelockert. Grundlage für sein Schaffen war die SIESMAYERsche Gärtnerei in der Schlossstraße in Bockenheim, der ein eigenes Kapitel gewidmet ist.

Im Anschluss werden einige große und kleine Gärten, die SIESMAYER geschaffen hat, vorgestellt, darunter beispielsweise der Bad Nauheimer Kurpark, der Stadtpark in Mainz oder der Park Rauischholzhausen bei Marburg. Gemäß der überregionalen Bedeutung des Gartens ist dem Palmengarten ein relativ langes Kapitel gewidmet. Der Leser trifft hier auf bekannte historische Darstellungen des alten Gesellschaftshauses mit dem Blumenparterre und den Teppichbeeten sowie des Steingartens mit Schweizer Haus und Wasserfall. HeINRICHS Sohn PHILIPP wurde sein Nachfolger, der wie sein Vater Spuren in der Geschichte der Gartenkunst hinterlassen hat. Auch ihm und seinen Gärten ist deshalb ein Kapitel am Schluss des Bandes gewidmet.

Informationen und die vielen historischen Abbildungen machen „SIESMAYERs Gärten“ für alle, die an historischen Gärten interessiert sind, sehr lesenswert.

HILKE STEINECKE 